parameters of a marginal structural model. In one of our most sophisticated conceptualizations of family structure effects, we found that recent change in family structure had a statistically significant effect on the odds of externalizing behavior: Odds Ratio $(95 \%$ Confidence Interval) $=2.95$ (1.73-5.02) . Overall, our substantive findings were tempered by methodological caveats, which have important implications for future studies in the area and for broader issues related to data collection, study design, and analysis.

1211

\section{PSYCHOMOTOR AND EMOTIONAL DEVELOPMENT AT 12 MONTHS OF VERY PRETERM INFANTS WITH BRONCHOPULMONARY DYSPLASIA}

C. Borradori Tolsa ${ }^{1}$, L. van Hanswijck de Jonge ${ }^{2}$, N. Langerock ${ }^{3}$, M. Monnier ${ }^{4}$, M. Bickle-Graz ${ }^{4}, \mathrm{~K}$. Barisnikov $^{3}$, P.S. Hüppi ${ }^{2}$

${ }^{1}$ Department of Child and Adolescent, ${ }^{2}$ Division of Development and Growth, ${ }^{3}$ Child Clinical Neuropsychology Unit, Geneva, ${ }^{4}$ Pediatrics, Children's Hospital, Lausanne, Switzerland

Cognitive, behavioral and emotional problems have been extensively reported for preterm children. BPD has been outlined in many studies as having an additional deleterious effect on early development. Our aim was to examine neurodevelopment and emotional regulation (ER) in preterm infants with BPD within the first year of life.

38 preterm infants (GA: $27.3 \pm 1.01 . w k s)$ were seen at 12 months corrected age, of which 19 have had BPD. Four components of the Laboratory Temperament Assessment Battery (Lab TAB) were administered in addition to the Bayley scales (MDI/ PDI). ER was derived from scores on the two trials of the anger evaluation of the Lab TAB.

By age of 12 months, mean PDI were lower in BPDgroup $(p<0.05)$ compared to non- BPD-group. No significant difference between groups was found for the MDI. Correlation analysis revealed a significant negative correlation between ER on the first anger trial and BW $(p<0.01)$ as well as with $G A(p<0.05)$, only for the BPD- group. These findings were no longer significant in the second trial. However, a trend indicated an improvement of ER over the two trials in the non-BPD group but not in the BPDgroup.
These results indicate that in children born preterm with BPD a higher BW as well as GA are protective factors allowing for better ER. These results add to the breadth of the literature indicating not only motor and intellectual development to be influenced by BPD at a young age but also the capacity to regulate emotions.

\section{2}

\section{THE PERINATAL MORTALITY IN LETO'} MATERNITY HOSPITAL NICU DURING 20072009, AND SURVIVAL OF NEWBORN INFANTS WITH BW $\leq 1500$ GRAMS

\section{A. Charitou, G. Kandili, A. Kleanthous, E. Siokou, N. Gkezerli \\ N.I.C.U., LETO Maternity Hospital, Athens, Greece}

Background and aims: Recording the survival of newborns in any Maternity Hospital is of utmost importance, as it contributes to the understanding of the level of perinatal care in Greece.

The aim is to present evidence about perinatal mortality and survival rate of very low birth weight (VLBW) neonates (birth weight (BW) $\leq 1,500$ grams) at the NICU at Leto Maternity Hospital.

Methods: Neonates transferred to other hospitals, admissions in Leto's NICU, perinatal mortality and neonatal survival (BW $\leq 1,500$ grams), were recorded and documented during 2007 to 2009.

Results: In 2007, 744 neonates admitted in the $\mathrm{NICU}, 37$ with $\mathrm{BW} \leq 1,500$ grams ( 11 with $\mathrm{BW}=500$ 1,000 grams and 26 with $B W=1,001-1,500$ grams). Five were transferred to other hospitals (three for financial reasons and two needed surgical operation treatment). In 2008, 908 neonates admitted in the $\mathrm{NICU}, 40$ with $\mathrm{BW} \leq 1,500$ grams ( 17 with $\mathrm{BW}=500$ 1,000 grams and 23 with $B W=1,001-1,500$ grams). Six were transferred to other hospitals (three needed intestinal surgery treatment, and three with posthemorrhagic hydrocephalus). In 2009, 911 newborns admitted in the NICU, 45 with $\mathrm{BW} \leq 1,500$ grams (15 with $B W=500-1,000$ grams and 30 with $\mathrm{BW}=1,001-1,500$ grams). Four were transferred to other hospitals (two for financial reasons and two with posthemorrhagic hydrocephalus). 11 early fetal deaths were recorded $(3,3$, and 5 for each year respectively) but no late neonatal fatalities (Table 1). 


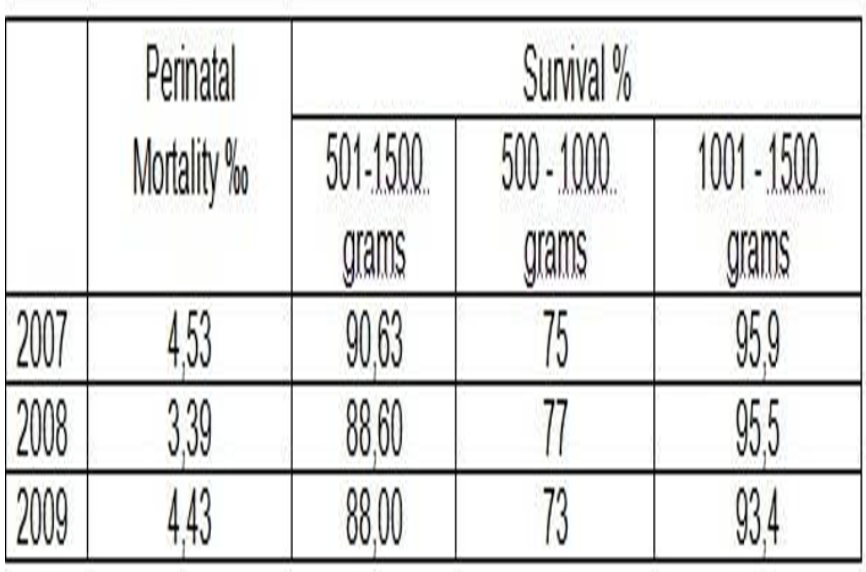

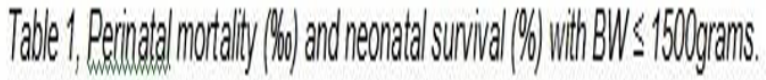

[Table 1]

Conclusions: The survival of VLBW neonates is considered to be equal to other European Union countries.

\section{3}

\section{THE IMPACT UPON PAEDIATRIC INTENSIVE CARE OF PREVIOUS ADMISSION TO THE NEONATAL INTENSIVE CARE UNIT - A 9 YEAR STUDY}

\author{
V.I. Astle ${ }^{1}$, H. Vyas ${ }^{1}$, K. Neal ${ }^{2}$, S.P. Wardle ${ }^{3}$, \\ S.E. Michael ${ }^{1}, \mathrm{H}$. Budge ${ }^{1}$
}

${ }^{1}$ Academic Child Health, School of Clinical Sciences, ${ }^{2}$ Epidemiology and Public Health, School of Community Health Sciences, ${ }^{3}$ Neonatal Intensive Care Unit, Nottingham University Hospitals, Nottingham, UK

Background and aims: Premature infants have more hospital admissions and stay longer on PICU ${ }^{1,2}$. As little is known about these PICU admissions, the aim of this study was to investigate the impact NICU infants have upon PICU.

Method: A retrospective cohort analysis was performed of $8791 \mathrm{NICU}$ admissions and 3307 PICU admissions at a University Hospital from April 2000-September 2009. NICU and PICU patients were compared to control groups to analyse: gestational age; sex; length of stay; diagnosis, survival and readmission rates.

Results: In the study period, 159 infants were admitted to both NICU and PICU. These infants had longer lengths of stay on NICU (Readmitted infants (R) (mean \pm sem): 35 days ( \pm 3.2$)$; Controls $(C): 21$ days $( \pm 1.9) P<0.001)$ and stayed longer on PICU (R: 8 ( \pm 2.2$) ; \mathrm{C}: 4( \pm 0.6)$ days $P<0.01)$. Long NICU stay predicted readmission to PICU. Premature infants (<33 weeks gestation) had a $20 \%$ greater risk of later being admitted to PICU than infants born in the hospital catchment area who were not premature. Although $4 \%$ of premature infants were later admitted to PICU, they did not have increased mortality on PICU.

Conclusion: NICU infants have increased length of stay on PICU, with a resultant cost impact, but not increased mortality. These data may be used to inform health professionals and parents of the subsequent risks of PICU admission in babies discharged from NICU.

1. Slonim A et al. Crit Care Med 2000;28(3):84853.

2. Odetola F et al. Pediatr Crit Care Med 2007;8(4):343-7.

1214

\section{SURVIVAL AFTER FETOSCOPIC GUIDED} LASER OCCLUSION (FLOC) OF TWIN-TO-TWIN TRANSFUSION SYNDROME
C.P. Halvorsen ${ }^{1}$, A. Dellgren ${ }^{2}$, S. Ek ${ }^{3}$, C. Grunewald ${ }^{1}$, M. Norman ${ }^{3}$

${ }^{1}$ Dept of Clinical Science and Education, Karolinska Institutet at Södersjukhuset, ${ }^{2}$ Fetal Care Center, Karolinska University Hospital, ${ }^{3}$ Dept of Clinical Science, Intervention and Technology,

Karolinska Institutet and University Hospital, Stockholm, Sweden

Background and aim: Untreated twin-to-twin transfusion syndrome (TTTS) carries a mortality rate of approximately $80 \%$. To improve survival, fetoscopic laser occlusion (FLOC) treatment of placental anastomoses has been introduced during the last decade in Sweden. We aimed to determine survival after introduction of this intervention.

Methods: Data from all TTTS pregnancies $(n=67)$ treated with FLOC from 2001-09 was assessed. In Sweden, FLOC is carried out at one center i.e. at Karolinska University Hospital, Stockholm. Rates of survival and intra-pair differences were analyzed, as well as maternal risk factors. The severity of TTTS in the cohort was graded according to Quintero (stage I $n=2$, stage II $n=30$, stage III $n=29$, stage IV 